\title{
Metas y satisfacción vital en adolescentes españoles y peruanos según sexo y nivel socioeconómico
}

\author{
Juan Francisco Díaz Morales ${ }^{1}$ \\ Universidad Complutense de Madrid
}

\author{
Patricia Martínez U.2 \\ Pontificia Universidad Católica del Perú
}

El presente estudio analiza el tipo de metas personales y la satisfacción vital en adolescentes españoles y peruanos según sexo y nivel socioeconómico. Se trabajó con 389 adolescentes españoles y con 285 peruanos, de ambos sexos y de diferentes niveles socioeconómicos. Los instrumentos utilizados fueron el Análisis de Metas Personales de Palys y Little (1983) y la Escala D-T (Delight-Terrible) de Andrews y Withey (1976) para la muestra española, y el Método de Inducción Motivacional de Nuttin (1985) junto con la Escala de Satisfacción con la vida de Pavot y Diener (1993) para la muestra peruana, que aunque no son equivalentes, permiten una comparación general. Los resultados muestran importantes semejanzas en las metas descritas por los adolescentes de ambos estudios, así como en la influencia de la variable sexo. Las principales diferencias entre los adolescentes españoles y peruanos se encuentran en la variable nivel socioeconómico.

Palabras clave: satisfacción con la vida, metas personales, adolescentes, sexo, nivel socioeconómico, cultura.

Goals and satisfaction with life in Spanish and Peruvian adolescents according to sex and socioeconomic level

This study analyzes the kind of personal goals and life satisfaction levels amongst Spanish and Peruvian adolescents according to their sex and socioeconomic level. 389 Spanish and $285 \mathrm{Pe}$ ruvian adolescents of both sexes from different socioeconomic level participated in this study. For the Spanish sample Palys and Little's (1983) Personal Goals Analysis and Andrews and Withey's (1976) D-T Scale (Delight-Terrible) were used; for the Peruvian sample the measurement instruments were Nuttin's (1985) Motivational Induction Method along with Pavot and Diener's (1993) Life Satisfaction Scale. Results show significant similarities in the goals described by both samples, as well as sex differences and socioeconomic differences.

Key words: satisfaction with life, personal goals, adolescents, sex, socioeconomic level, culture.

1 Doctor en Psicología, Profesor Ayudante de la Universidad Complutense de Madrid, España. Correo electrónico: juanfcodiaz@psi.ucm.es.

2 Doctora en Psicología, Profesora Asociada de la Pontificia Universidad Católica del Perú. Correo electrónico: pmartin@pucp.edu.pe. 

Diversos autores subrayan la importancia de la construcción de metas y proyectos para la comprensión de la conducta presente. La conducta del individuo se regula, en última instancia, de acuerdo con los proyectos que se construyen y que reflejan una concepción de sí mismo y del entorno. En este contexto, desarrollarse viene a ser transformarse en lo que se desea ser, y se constituye en un aspecto central del desarrollo humano (Nuttin, 1985).

Es en la etapa adolescente donde la construcción de metas adquiere una importancia fundamental, ya que se trata de una etapa de tránsito a la adultez. Es una etapa de cambios y de importantes consecuencias para etapas posteriores, definida por Castillo (1999) como la etapa del proyecto personal de vida, donde la tarea fundamental consiste en lograr formular metas claras con respecto al futuro.

Los tipos de metas que los jóvenes se plantean, especialmente si ellas tienen que ver con el desarrollo de las tareas propias de su periodo evolutivo, influyen en su bienestar subjetivo o satisfacción con la vida (Nurmi, 1991) y viceversa, cómo la gente evalúa su vida, en términos de su bienestar subjetivo, influye en el tipo de metas que ellos construyen más tarde. De acuerdo con la teoría de la auto-definición y auto-dirección del ciclo vital de Nurmi (1991) es predecible que aquellas metas personales relacionadas con las tareas de desarrollo propias de la edad y demandas sociales, provean una base para una socialización exitosa y por consiguiente aumenten el bienestar subjetivo. La segunda posibilidad es que la manera en que la gente evalúa su situación vital en términos del bienestar subjetivo influya en las metas que se plantean o construyen. Ambas posibilidades son comprobadas en el trabajo de Salmela-Aro y Nurmi (1997), quienes constatan un modelo bidireccional: los jóvenes que se interesan por 
metas que reflejan en mayor medida tareas normativas propias de su periodo de edad, particularmente aquellas relacionadas con su futura familia, trabajo y estudios, experimentan un aumento del bienestar dos años más tarde. Salmela-Aro y Nurmi lo denominan ciclo acumulativo. Los jóvenes están preocupados por metas relacionadas con el desarrollo de tareas vitales que tienen que ver con la educación, familia y ocupación.

Muchos de los trabajos que han analizado el tema coinciden en las categorías de agrupación de las metas vitales, lo cual es esperable considerando el periodo vital al que nos estamos refiriendo. SalmelaAro y Nurmi (1997) defienden que estas metas dirigen los planes de vida futuros de las personas, sus transiciones y los eventos vitales por los que atraviesan.

Son pocos los estudios en relación a cómo influye el nivel socioeconómico sobre el contenido de las metas e intereses de los adolescentes (Nurmi, 1991). Los resultados indican que los adolescentes asignados a la clase baja enfatizan más su pensamiento futuro en el trabajo del que vivirán, mientras que los adolescentes de la clase media tienden a estar más interesados en la educación, desarrollo de carrera y actividades de ocio y tiempo libre. Otros trabajos encuentran que los adolescentes de clase media tienen una extensión de futuro mayor que los adolescentes de clase baja. Una explicación de estos datos es que los adolescentes de clase media-alta anticipan sus tareas vitales en un marco temporal mayor que los adolescentes de clase baja seguramente debido a su posición económica y social. Como afirma Nuttin (1985) la menor extensión temporal de los adolescentes de clase baja se debe a una evaluación realista de su situación más que a deficiencias en el pensamiento relacionado con el futuro. Los trabajos en relación a la planificación del futuro encuentran que los adolescentes de un status socioeconómico mayor planifican su futuro en mayor medida que los adolescentes de un status socioeconómico más bajo. 
Metas y satisfacción vital en adolescentes españoles y peruanos...

En cuanto a las diferencias según sexo, tradicionalmente los varones participan más activamente en su educación y en el trabajo, mientras que las mujeres están más ocupadas $\mathbf{u}$ orientadas a las actividades domésticas y a la familia (Nurmi, 1991; Salmela-Aro \& Nurmi, 1997). Los hombres se plantean metas relacionadas con los aspectos materiales de la vida y las mujeres en relación con su futura familia. En el estudio de Gillispie y Allport (en Nuttin, 1985) las mujeres esperan ser más felices con el matrimonio, mientras que los hombres desean más que las mujeres tener buena salud. Según Lueptow (1984) las mujeres y hombres responden a las preguntas en relación con las metas vitales de un modo estereotipado: las mujeres valoran la religión, hacer contribuciones a la sociedad y la familia, mientras que los hombres valoran el éxito y estatus social. No hay diferencias de sexo en relación a cuán importante es el trabajo.

Algunos autores que encuentran diferencias en la extensión temporal de los adolescentes atribuyen estas diferencias al contenido de las metas de uno u otro sexo: los objetivos de las mujeres se sitúan a más corto plazo que las metas de los hombres (familia, matrimonio, hijos versus status, prestigio, poder y aspectos materiales de la vida). Aunque ambos dan igual importancia a las metas relacionadas con el trabajo, para las mujeres el marco temporal es más corto que para los hombres. Kammer (1985) señala que un $25 \%$ de las mujeres de su estudio son incapaces de describir algún plan o proyecto educativovocacional. Su hipótesis es que evitan el conflicto familia-trabajo situando sus metas familiares por encima del trabajo. Cuando evalúan la perspectiva de los adolescentes en relación a su plan vocacional, encuentran que las mujeres disponen de un mayor repertorio de alternativas de estudio (sobre todo con estudios o trabajos que impliquen el trabajo con los demás), mientras que los hombres tienen una línea bastante definida sobre lo que quieren hacer. Estas diferencias reproducen las expectativas asignadas al rol sexual e incluso a los 13 años las mujeres anticipan un mayor número de alternativas laborales que les permitan combinar trabajo y familia. Los hombres tienen una línea 
más definida pues no prevén interrupciones en su desarrollo de carrera. Del mismo modo, otros trabajos encuentran que los hombres adolescentes esperan conseguir un trabajo a tiempo completo, mientras que las mujeres adolescentes esperan un trabajo de medio tiempo (Post, Williams \& Brubaker, 1996).

A partir de lo anterior, resulta interesante investigar si estas predicciones se confirman en dos países con dos culturas diferentes, España y Perú. Nuestro propósito es analizar qué tipo de metas se plantean los adolescentes de diferente sexo y nivel socioeconómico y si, considerando estas diferencias, las metas son diferentes según el país.

\section{ESTUDIO 1: ADOLESCENTES ESPAÑOLES}

\section{Metodología}

\section{Participantes}

Los participantes de este estudio fueron 389 adolescentes estudiantes de Enseñanza Secundaria Obligatoria (E.S.O.), con una edad comprendida entre los 15 y 18 años. La media de edad del grupo fue de 16.2 años $(D E=1.09)$. La media de edad de las mujeres fue de $16.1(D E=1.07)$ y de los hombres de $16.3(D E=1.12)$. El grupo estuvo compuesto por $229(58.8 \%)$ mujeres y $160(41.2 \%)$ hombres. La edad de los sujetos se corresponde con el último curso de educación secundaria obligatoria (15 a 16 años) y los estudios de bachillerato LOGSE (17 a 18 años). Los adolescentes pertenecían a tres institutos de enseñanza secundaria de la Comunidad de Madrid.

El nivel socioeconómico (NSE) fue calculado a partir del índice de Estatus Socioeconómico de Fernández (1980). Se ha tenido en cuenta si los dos padres trabajaban, así como el tipo de trabajo de los mismos. Si el trabajo de los padres suponía un nivel de calificación 
Metas y satisfacción vital en adolescentes españoles y peruanos...

medio-alto (licenciatura, diplomatura, etc.) y estaba relacionado con el empleo en empresas como ejecutivos o profesionales cualificados, se consideraba un nivel socioeconómico alto $(32,2 \%)$; ocupaciones relacionadas con pequeños negocios autónomos o empresas familiares por cuenta propia se consideraba un nivel socioeconómico medio $(31,9 \%)$; y si el trabajo desempeñado por los padres no exigía calificación, como obreros o dependientes, se consideraba un nivel socioeconómico bajo $(32,2 \%)$. Según este criterio, la distribución de la muestra ha quedado configurada en tres grupos, como aparece en el Cuadro 1.

\section{Cuadro 1}

Distribución según sexo y NSE de los adolescentes españoles

\begin{tabular}{lccc}
\hline NSE & Mujeres & Varones & Total \\
\hline NSE bajo & 88 & 42 & 130 \\
NSE medio & 76 & 53 & 129 \\
NSE alto & 65 & 65 & 130 \\
\hline Total & 229 & 160 & 389 \\
\hline
\end{tabular}

\section{Instrumentos}

1. Escala de Satisfacción Vital en diferentes áreas

Basada en la escala D-T (Delight-Terrible) de Andrews y Withey (1976), incluye la evaluación de diferentes dominios vitales. Los dominios o áreas vitales utilizados han sido: estudios, familia, tiempo libre, amigos, compañeros de estudio, salud física, salud psicológica, nivel de vida en general, vida afectiva y con uno mismo. El adolescente tiene que valorar el grado de satisfacción en cada escala con un formato tipo Likert de 7 puntos. Se obtiene una puntuación total que es el resultado de la satisfacción (total) en todas las áreas. Asimismo, se le solicita que ordene las áreas vitales de mayor a menor grado de importancia. Las áreas elegidas pueden considerarse relevantes para la etapa adolescente tal y como se han estudiado en otros estudios 
previos (Castro Solano, 2000; Castro Solano \& Díaz Morales, 2002; Díaz Morales, 2000; Díaz Morales \& Sánchez-López, 2002).

\section{Análisis de Metas Personales (Palys \& Little, 1983)}

Consiste en una adaptación de la metodología propuesta originalmente por Little (1983) en relación al análisis de proyectos personales y utilizada, con algunas modificaciones, por autores como Emmons (1986), Pervin (1989) y Cantor (1992). Los adolescentes tienen que enumerar una serie de metas personales que sean significativas en su momento del ciclo vital y que pretendan realizar en un futuro cuya extensión temporal no es prefijada por el evaluador. Cada adolescente enumeró hasta diez metas personales y posteriormente cada meta fue evaluada en las siguientes dimensiones: apoyos, dificultades, progreso, conflicto, tiempo adecuado, control y conocimiento de otros. Estas dimensiones son propuestas en la revisión de Little (1999) como las más relevantes para el análisis de las metas personales. Se ha utilizado una escala tipo Likert de 7 puntos, lo que permite efectuar comparaciones entre los diferentes individuos (aproximación nomotética) sin perder lo único y personal de cada uno (aproximación ideográfica). Los análisis de fiabilidad en población adolescente española son aceptables. Las dimensiones en las que son evaluadas las metas personales muestran una alta consistencia interna con valores que oscilan desde $\mathrm{a}=0,67$ para la dimensión dificultades hasta $\mathrm{a}=$ 0,82 para la dimensión conflicto (Díaz Morales, 2001). La validez del instrumento de la evaluación de las metas personales mediante la valoración de cada una de las metas que enumeran los adolescentes en determinadas dimensiones, ha sido estudiada considerando su relación con otros constructos psicológicos (Little, 1999; Sheldon \& Elliot, 2000). La estrategia de análisis más utilizada es la reducción del número de dimensiones de las metas a varios factores. Esta estrategia es utilizada cuando el número de dimensiones en que son evaluadas las metas es muy elevado (por ejemplo, las 15 dimensiones de Little, 1983 y Emmons, 1986). 
Metas y satisfacción vital en adolescentes españoles y peruanos...

El procedimiento de Análisis de las Metas Personales de Little (1983) ha sido utilizado con éxito en estudios previos. Así, por ejemplo, en el análisis de la configuración de perfiles de metas personales y perfiles de satisfacción en diferentes áreas vitales de adolescentes españoles y argentinos (Castro Solano \& Díaz Morales, 2002); en la predicción de la satisfacción vital a través de los estilos de personalidad y las metas personales (Díaz Morales \& Sánchez-López, 2001); e incluso, en el análisis de las metas deportivas de jugadores de fútbol (Díaz Morales \& Naveira, 2001).

\section{Procedimiento}

La aplicación de las pruebas se realizó de forma colectiva, cumpliendo las normas deontológicas oportunas y garantizando el anonimato de los participantes. Se ha utilizado el Análisis de Varianza (ANOVA) para calcular la diferencia de medias en satisfacción según el sexo y el nivel socioeconómico (NSE). También se ha empleado el estadístico Chi Cuadrado $\left(c^{2}\right)$ para calcular la asociación entre variables nominales como el sexo y el NSE, así como el Coeficiente de Contingencia (C) como indicador del grado de relación entre ellas.

\section{Resultados} ñoles?

¿Qué categorías de metas predominan en los adolescentes espa-

Hemos establecido las mismas categorías que otros autores (Nurmi 1989; Nurmi, Poole \& Seginer, 1995). Se han creado siete categorías a partir de un conjunto más amplio de 15 categorías previas (Díaz Morales, 2000). Las siete «supracategorías» son las siguientes:

- Metas intrapersonales o metas relacionadas con uno mismo: esta supracategoría agrupa a las categorías intrapersonal, independencia, 
salud y cambios o sucesos vitales. Todas ellas se refieren a aspectos de la propia personalidad.

- Metas relacionadas con los amigos: formada por la categoría amigos.

- Metas relacionadas con la familia/pareja: formar una familia propia, respecto a la familia actual y en relación con la pareja.

- Metas relacionadas con el trabajo: formada por la categoría trabajo.

- Metas relacionadas con los estudios: estudios a corto plazo, a largo plazo definidos e indefinidos y cursos/formación complementaria.

- Metas relacionadas con hobbies, ocio, deportes: categoría hobbies, ocio, etc.

- Metas relacionadas con cosas materiales o posesiones: está formada únicamente por la categoría cosas materiales.

Hemos diferenciado las metas referidas al trabajo de las metas relacionadas con los estudios. En algunos trabajos (Salmela-Aro \& Nurmi, 1997) se consideran metas relacionadas con el logro o realización, mientras que otros autores (Little, 1983) diferencian entre metas relacionadas con el trabajo de las relacionadas con los estudios o "académicas". Se ha seguido la diferenciación de Little, pues la frecuencia de ambas categorías es semejante y dado el momento vital de los sujetos puede ser una categoría relevante: algunos adolescentes pueden centrarse en buscar un trabajo, mientras que otros pueden seguir estudios medios o superiores. La clasificación que hemos realizado refleja las tareas evolutivas propias de este periodo de edad, pues la mayoría de los autores encuentran que las metas relacionadas con los estudios, trabajo y familia son las tareas de desarrollo que los adolescentes de esta edad anticipan y que desarrollarán en un futuro (Dannefer, 1984; Nurmi, 1991; Nuttin, 1985). 
Metas y satisfacción vital en adolescentes españoles y peruanos...

\section{Cuadro 2}

Categorías de metas más relevantes para la muestra de estudio

\begin{tabular}{lcc}
\hline Metas & Frecuencia & $\%$ \\
\hline Estudios & 396 & 96,8 \\
Familia & 280 & 68,5 \\
Trabajo & 249 & 60,9 \\
Personalidad & 234 & 57,2 \\
Amigos & 186 & 45,5 \\
Posesiones & 133 & 32,5 \\
Hobbies & 105 & 25,7 \\
\hline
\end{tabular}

¿Hay diferencias en el tipo de metas según el sexo en los adolescentes españoles?

Hemos analizado el porcentaje de varones y mujeres adolescentes que enumeran metas relacionadas con las siete supracategorías anteriormente mencionadas. Encontramos más mujeres $(69,9 \%)$ que hombres $(30,1 \%)$ que se plantean metas relacionadas con los amigos $\left(\chi^{2}=18,38 p<0,001 ; \mathrm{C}=0,20 p<0,001\right)$. En contraste, más hombres que mujeres, $67,5 \%$ versus $32,5 \%$, enumeran metas relacionadas con las cosas materiales o posesiones $\left(\chi^{2}=28,23 p<0,001 ; \mathrm{C}=0,25 p<\right.$ $0,001)$. En el resto de las supracategorías no aparecen diferencias significativas. Es decir, las mujeres adolescentes se plantean metas relacionadas con los amigos en mayor medida que los hombres y enumeran menos metas relacionadas con las posesiones o cosas materiales.

¿Hay diferencias en el tipo de metas según el NSE en los adolescentes españoles?

Aparecen relaciones entre las metas relacionadas con los hobbies y aficiones y bajo nivel socioeconómico. Hay más adolescentes con alto nivel socioeconómico $(40,4 \%)$ que enumeran metas relacionadas con los hobbies y aficiones, frente a los adolescentes de nivel socioeconómico 
medio $(35,6 \%)$ y bajo $(24,0 \%)\left(\chi^{2}=6,04 ; \mathrm{gl}=2 ; p<0,05 ; \mathrm{C}=0,12 p<\right.$ $0,05)$.

¿Hay diferencias en el tipo de metas y satisfacción según el sexo y el NSE en los adolescentes españoles?

Para analizar las relaciones conjuntas entre el nivel socioeconómico, el sexo, la categoría de metas y la satisfacción con la vida, se han creado seis grupos de adolescentes a partir de la combinación del NSE (alto, medio y bajo) y el sexo. En primer lugar, se ha calculado para cada grupo el nivel de satisfacción. Las medias y desviaciones estándar aparecen en el Cuadro 3. Se ha realizado un ANOVA entre los grupos que indica ausencia de diferencias estadísticamente significativas $[\mathrm{F}(5,373)=2.19, p=0.054]$. Las diferencias en satisfacción entre los grupos son no significativas.

Como aparece en el Cuadro 3, las mujeres con bajo NSE tienden a una mayor puntuación en satisfacción, mientras que los varones de alto NSE obtienen el menor nivel de satisfacción, pero las diferencias no son significativas.

\section{Cuadro 3}

Satisfacción vital en cada grupo definido por el sexo y el NSE

\begin{tabular}{lccc}
\hline \multicolumn{1}{c}{ Grupos } & $M$ & $D E$ & $N$ \\
\hline Mujeres y alto NSE & 53,52 & 8,95 & 64 \\
Mujeres y medio NSE & 53,04 & 8,93 & 71 \\
Mujeres y bajo NSE & $\mathbf{5 6 , 4 1}$ & 7,37 & 86 \\
Varones y alto NSE & $\mathbf{5 2 , 5 4}$ & 9,72 & 65 \\
Varones y medio NSE & 54,73 & 8,51 & 52 \\
Varones y bajo NSE & 55,39 & 7,00 & 41 \\
\hline Total & 54,28 & 8,57 & 379 \\
\hline
\end{tabular}

Posteriormente, se ha considerado la presencia y ausencia de metas en cada categoría en cada uno de los grupos mediante la prueba 
Metas y satisfacción vital en adolescentes españoles y peruanos...

Chi Cuadrado. Sólo aparecen diferencias en las categorías amigos $\left[\chi^{2}\right.$ $(5)=20.85, p<0.001 ; C=0.22, p<0.001]$ y posesiones $\left[\chi^{2}(5)=31.05\right.$, $p<0.001 ; \mathrm{C}=0.27, p<0.001]$. Las mujeres con bajo NSE se plantean un mayor número de metas relacionadas con los amigos (Cuadro 4). Por el contrario, los varones de alto NSE se plantean un mayor número de metas relacionadas con las posesiones o cosas materiales (Cuadro 5).

\section{Cuadro 4}

Frecuencia de metas relacionadas con los amigos en los seis grupos

\begin{tabular}{lccc}
\hline Grupos & Ausencia & Presencia & Total \\
\hline Mujeres y alto NSE & 27 & 38 & 65 \\
Mujeres y medio NSE & 33 & 43 & 76 \\
Mujeres y bajo NSE & 41 & 47 & 88 \\
Varones y alto NSE & 44 & 21 & 65 \\
Varones y medio NSE & 33 & 20 & 53 \\
Varones y bajo NSE & 30 & 12 & 42 \\
\hline Total & 208 & 181 & 389 \\
\hline
\end{tabular}

$\chi^{2}=20.85, p<0.001 ; \mathrm{C}=0.22, p<0.001$

\section{Cuadro 5}

Frecuencia de metas relacionadas a posesiones materiales en los seis grupos

\begin{tabular}{lccc}
\hline Grupos & Ausencia & Presencia & Total \\
\hline Mujeres y alto NSE & 47 & 18 & 65 \\
Mujeres y medio NSE & 65 & 11 & 76 \\
Mujeres y bajo NSE & 67 & 21 & 88 \\
Varones y alto NSE & 35 & 30 & 65 \\
Varones y medio NSE & 30 & 23 & 53 \\
Varones y bajo NSE & 20 & 22 & 42 \\
\hline Total & 264 & 125 & 389 \\
\hline
\end{tabular}

$\chi^{2}=31.05, p<0.001 ; \mathrm{C}=0.27, p<0.001$ 
En resumen, las adolescentes de bajo nivel socioeconómico muestran una mayor satisfacción (no significativa). Este grupo se plantea un mayor número de metas relacionadas con los amigos. Por el contrario, encontramos que los varones adolescentes obtienen el nivel de satisfacción vital más bajo (no significativo). Este grupo se plantea en mayor medida un mayor número de metas relacionadas con las posesiones y cosas materiales. En el resto de categorías de metas, no aparecen diferencias significativas.

\section{ESTUDIO 2: ADOLESCENTES PERUANOS}

\section{Metodología}

\section{Participantes}

El grupo total estuvo constituido por 285 jóvenes, entre los 16 y los 25 años, de los cuales 143 se encontraban en el último año de instrucción secundaria y 142 se encontraban en la universidad. El NSE se determinó en función a tres indicadores definidos en base a los estudios de Apoyo (2002): el tipo de centro de estudios (Nacional o Privado), el nivel de instrucción de los padres y el distrito de residencia. Esto permitió diferenciar dos grupos, NSE alto y medio alto por un lado, y NSE bajo y medio bajo, por otro.

Ambos grupos de edad presentan características similares, no se encuentran diferencias significativas en ninguna de las variables estudiadas, por lo que se trabajó con el grupo como conjunto.

\section{Cuadro 6}

Distribución según sexo y NSE de los adolescentes peruanos

\begin{tabular}{cccc}
\hline NSE & Mujeres & Varones & Total \\
\hline Alto & 70 & 72 & 142 \\
Bajo & 72 & 71 & 143 \\
Total & 142 & 143 & 285 \\
\hline
\end{tabular}


Metas y satisfacción vital en adolescentes españoles y peruanos...

\section{Instrumentos}

1. Escala de Satisfacción con la Vida (SWLS)

Se trata de una escala destinada a evaluar la satisfacción global con la vida, desarrollada por Pavot y Diener (1993). Consiste en un conjunto de cinco afirmaciones frente a las cuales las personas deben indicar su nivel de acuerdo o desacuerdo en una escala de siete puntos. El puntaje total de la prueba oscila entre 5 y 35 , que puede interpretarse tanto en términos absolutos como relativos. Un puntaje de 20 representa un punto neutral e indica que estas personas no están ni satisfechas ni insatisfechas con su vida. Del 21 al 25 están ligeramente satisfechas, del 15 al 19 ligeramente insatisfechas, del 26 al 30 satisfechas y del 10 al 14 insatisfechas. Los estudios normativos realizados con la prueba en diferentes grupos y nacionalidades (Estados Unidos, Francia, China, Rusia, etc.) indican que las medias en todos estos estudios con poblaciones normales se ubican en la categoría de ligeramente satisfechos o satisfechos. En muestras clínicas, sin embargo, los puntajes tienden a ser menores (Pavot \& Diener, 1993), lo que constituye una prueba de su validez.

La validez de constructo de la SWLS ha sido analizada recientemente en grandes muestras y comparando diferentes poblaciones (Arrindell, Heesink \& Feij, 1999; Shevlin, Brunsden \& Miles, 1998). Se ha trabajado también en población argentina (Castro Solano, 2000), española (Díaz Morales, 2001) y peruana (Martínez, 2004) mostrando en todos los casos buenas propiedades psicométricas. Numerosos estudios con la prueba demuestran una alta consistencia interna (Alpha de .87) y una estabilidad temporal moderada. Por otro lado, el análisis factorial de la prueba muestra la presencia de un solo factor que explica el $66 \%$ de la varianza, que además se mantiene en diferentes estudios. Para el presente estudio se realizó un análisis factorial de la prueba que confirma los estudios reportados, se encontró un sólo factor que explicaba el $57.63 \%$ de la varianza total. En el 
análisis de la consistencia interna de la prueba mediante el Alpha de Cronbach se encontró un Alpha total de .81.

\section{Método de Inducción Motivacional (MIM)}

Se trata de una prueba destinada a identificar los objetos motivacionales propios de cada individuo y situarlos en una escala temporal con el fin de medir su extensión (Nuttin, 1985). Para fines del presente estudio, únicamente trabajamos las metas u objetos motivacionales. La prueba consiste en un cuadernillo con tarjetas que contienen frases o inductores en primera persona que inducen al examinado a expresar sus metas, deseos y temores. A partir de ello se pueden identificar categorías motivacionales generales a las cuales pertenece cada una de estas metas. La versión original de la prueba consta de 60 ítemes, 40 de los cuales son positivos y 20 negativos. A partir de esto se han desarrollado versiones reducidas; la que se usó para la presente investigación constó de 30 ítemes, 20 de los cuales son positivos y 10 negativos. La codificación de la prueba se realiza a partir de los códigos desarrollados por Nuttin y Lens (en Nuttin, 1985). De acuerdo a los planteamientos del autor, sólo se trabajó con la primera respuesta para cada uno de los inductores, de tal manera que en cada caso se trabajó con un total de 30 respuestas.

Para el análisis de contenido se proponen diez categorías generales y un conjunto de subcategorías que incluyen además combinaciones particulares de contenidos que deben adecuarse a los objetivos de cada investigación. Las categorías y subcategorías usadas en el presente estudio fueron:

1. Sí Mismo: metas relativas a la personalidad

- Autoconcepto: percepción de sí mismo

- Autonomía: autonomía personal

2. Autorrealización y Realización: desarrollo del sí mismo a través de actividades productivas 
Metas y satisfacción vital en adolescentes españoles y peruanos...

- Vinculados a Estudios

- Vinculados a Trabajo

3. Contacto Interpersonal

- Contacto Altruista

- Relación con Padres

- Relación con Amigos

4. Exploración: motivaciones cognitivas, de conocimiento

5. Posesiones: deseo de poseer o adquirir objetos

6. Diversión: actividades de recreación o placer

Nuttin (1985) reporta diferentes estudios sobre la validez de la prueba, que demuestran su sensibilidad para identificar los objetos motivacionales de grupos específicos. Los estudios de confiabilidad en la codificación de la prueba, reportados por el autor, se realizaron con dos jueces independientes. El nivel de coincidencia en la categorización de las respuestas entre ambos jueces, en distintos estudios alcanzó entre el $80 \%$ y el $90 \%$ de acuerdos. En el Perú se han realizado dos estudios con el MIM en adolescentes escolares (Herrera, 2002; Martínez, 2002, 2004), en los que se confirma la utilidad de la prueba para la medición de la Perspectiva Temporal Futura.

\section{Procediniento}

Las pruebas del grupo de adolescentes escolares fueron aplicadas de manera colectiva en sus respectivas aulas de clase. Las del grupo de universitarios se aplicaron en pequeños grupos de diferentes especialidades. En ambos casos las pruebas fueron anónimas y los participantes aceptaron participar voluntariamente en la investigación. Los estadísticos utilizados fueron los mismos que los de la muestra española, es decir, Análisis de Varianza (ANOVA) para la comparación de los niveles de satisfacción en los diferentes grupos y Chi Cuadrado $\left(\mathrm{c}^{2}\right)$ para el análisis de las metas. 


\section{Resultados}

¿Qué categorías de metas predominan en los adolescentes peruanos?

Las metas que predominan en el grupo se presentan en el Cuadro 7. Aquí se puede observar que las metas relativas a Sí Mismo y Contacto Interpersonal están presentes prácticamente en todos los participantes. Además de ellas, las que se presentan con mayor frecuencia son: Familia y Pareja, Trabajo y Estudios.

¿Hay diferencias en el tipo de metas según el sexo en adolescentes peruanos?

Al comparar estas categorías por sexo encontramos diferencias significativas entre varones y mujeres en cuatro de ellas. Así, en los hombres encontramos mayor presencia de metas relativas a Diversión, y en las mujeres mayor presencia de metas relativas a la Familia y Pareja, a los Padres y al Contacto Altruista (Cuadro 8).

\section{Cuadro 7}

Categorias de metas más relevantes para los participantes

\begin{tabular}{lcc}
\hline Metas & Frecuencia & $\%$ \\
\hline Sí Mismo & 284 & 99.6 \\
Contacto Interpersonal & 283 & 99.3 \\
Familia/Pareja & 248 & 87.0 \\
Trabajo & 208 & 73.0 \\
Estudios & 200 & 70.2 \\
Autonomía & 141 & 49.5 \\
Padres & 122 & 42.8 \\
Diversión & 118 & 41.4 \\
Exploración & 90 & 31.6 \\
Autoconcepto & 86 & 30.2 \\
Amigos & 77 & 27.0 \\
Posesiones & 69 & 24.2 \\
Contacto Altruista & 67 & 23.5 \\
\hline
\end{tabular}


Metas y satisfacción vital en adolescentes españoles y peruanos...

\section{Cuadro 8}

Diferencias en la presencia de metas de acuerdo al sexo

\begin{tabular}{|c|c|c|c|c|c|c|}
\hline \multirow[t]{2}{*}{ Metas } & \multicolumn{2}{|c|}{ Hombres } & \multicolumn{2}{|c|}{ Mujeres } & \multirow[t]{2}{*}{$\chi^{2}$} & \multirow[t]{2}{*}{$p$} \\
\hline & $N$ & $\%$ & $N$ & $\%$ & & \\
\hline Familia/Pareja & 118 & 82.5 & 130 & 91.5 & 5.145 & $.023 *$ \\
\hline Diversión & 72 & 50.3 & 46 & 32.4 & 9.468 & $.002 * *$ \\
\hline Contacto Altruista & 25 & 17.5 & 42 & 29.6 & 5.796 & $.016^{*}$ \\
\hline Padres & 51 & 35.7 & 71 & 50.0 & 5.981 & $.014 *$ \\
\hline
\end{tabular}

${ }^{*} p<.05, * * p<.01$

¿Hay diferencias en el tipo de metas según el NSE en adolescentes peruanos?

Del mismo modo, en la comparación por nivel socioeconómico, encontramos diferencias significativas en la presencia de metas relativas a los Estudios, a los Padres y al Contacto Altruista, en todos los casos con una frecuencia superior en el NSE bajo (Cuadro 9).

\section{Cuadro 9}

Diferencias en la presencia de metas de acuerdo al NSE

\begin{tabular}{lccccccc}
\hline \multirow{2}{*}{ Metas } & \multicolumn{2}{c}{ NSE Alto } & & \multicolumn{2}{c}{ NSE Bajo } & $\chi^{2}$ & $p$ \\
\cline { 2 - 3 } & $N$ & $\%$ & & $N$ & $\%$ & & \\
\hline Estudios & 92 & 64.8 & & 108 & $\mathbf{7 5 . 5}$ & 3.924 & $.048^{*}$ \\
Padres & 50 & 35.2 & & 72 & $\mathbf{5 0 . 3}$ & 6.669 & $.010^{*}$ \\
Contacto Altruista & 22 & 15.5 & & 45 & $\mathbf{3 1 . 5}$ & 10.112 & $.001^{* *}$ \\
\hline
\end{tabular}

${ }^{*} p<.05,{ }^{* *} p<.01$

¿Hay diferencias en satisfacción vital según el sexo y NSE en adolescentes peruanos?

Al combinar el sexo y nivel socioeconómico y los niveles de satisfacción en el grupo no encontramos diferencias significativas, pero 
sí una tendencia a un mayor nivel de satisfacción en las mujeres de NSE alto. Asimismo, los menores niveles de satisfacción se encuentran también en las mujeres, pero de NSE bajo.

\section{Cuadro 10}

Satisfacción en cada grupo definido por el sexo y el NSE

\begin{tabular}{lccc}
\hline Grupos & $M$ & $D E$ & $N$ \\
\hline Hombres y NSE alto & 21.85 & 5.96 & 72 \\
Mujeres y NSE alto & $\mathbf{2 2 . 0 1}$ & 5.92 & 70 \\
Hombres y NSE bajo & 21.83 & 5.52 & 71 \\
Mujeres y NSE bajo & 20.60 & 5.10 & 72 \\
\hline Total & 21.57 & 5.63 & 285 \\
\hline
\end{tabular}

Finalmente, al combinar ambas variables (sexo y nivel socioeconómico), con las metas que describen los participantes, encontramos diferencias significativas en la misma línea de lo anterior, en las categorías de Estudios, Contacto Altruista y Relación con los Padres.

En el caso de las metas relativas a los Estudios (Cuadro 11), la mayor presencia de estos contenidos se encuentra en el NSE bajo, tanto en hombres como en mujeres. En las metas de Contacto Altruista (Cuadro 12), es en el grupo de mujeres de NSE bajo donde existe una mayor presencia de estas metas en relación a los otros grupos. Llama la atención, asimismo, su escasa presencia en los hombres de NSE alto. Por último, las metas relativas a los Padres o a la relación con ellos (Cuadro 13) se presentan predominantemente en las mujeres de NSE bajo, siendo significativamente más baja su presencia en los hombres de NSE alto. 
Metas y satisfacción vital en adolescentes españoles y peruanos...

\section{Cuadro 11}

Metas relativas a los Estudios de acuerdo al sexo y NSE

\begin{tabular}{lccc}
\hline Grupos & Presencia & Ausencia & $N$ \\
\hline Hombres y alto NSE & 42 & 30 & 72 \\
Mujeres y alto NSE & 50 & 20 & 70 \\
Hombres y bajo NSE & $\mathbf{5 4}$ & 17 & 71 \\
Mujeres y bajo NSE & $\mathbf{5 4}$ & 18 & 72 \\
\hline$\chi^{2}=6.851, p<.05$ & & &
\end{tabular}

\section{Cuadro 12}

Metas relativas al Contacto Altruista de acuerdo al sexo y NSE

\begin{tabular}{lccl}
\hline Grupos & Presencia & Ausencia & $N$ \\
\hline Hombres y alto NSE & 7 & 65 & 72 \\
Mujeres y alto NSE & 15 & 55 & 70 \\
Hombres y bajo NSE & 18 & 53 & 71 \\
Mujeres y bajo NSE & $\mathbf{2 7}$ & 45 & 72 \\
\hline$\chi^{2}=15.751, p<.001$ & & &
\end{tabular}

\section{Cuadro 13}

Metas relativas a los Padres de acuerdo al sexo y NSE

\begin{tabular}{lccc}
\hline Grupos & Presencia & Ausencia & $N$ \\
\hline Hombres y alto NSE & 20 & 52 & 72 \\
Mujeres y alto NSE & 30 & 40 & 70 \\
Hombres y bajo NSE & 31 & 40 & 71 \\
Mujeres y bajo NSE & $\mathbf{4 1}$ & 31 & 72 \\
\hline
\end{tabular}

$\chi^{2}=12.542, p<.01$

En resumen, las categorías que mayores diferencias presentan son Estudios, Contacto Altruista y Relación con los Padres, con una mayor frecuencia en los grupos de NSE bajo y en las mujeres. 


\section{Discusión}

Un primer tema importante se encuentra en la similitud de resultados en ambos estudios, a pesar de haberse utilizado instrumentos de evaluación diferentes. Si bien esto constituye una limitación metodológica importante para la comparación de resultados, el hecho de que todos los instrumentos hayan sido probados previamente en otros estudios demostrando su validez y confiabilidad, nos permite hacer ciertas inferencias con respecto a las semejanzas y diferencias encontradas en ambas poblaciones.

Así, en ambos estudios, es significativa la mayor frecuencia de metas relativas a la Familia y Pareja, a los Estudios y al Trabajo, que confirman los temas de preocupación centrales en los adolescentes, independientemente de la cultura (Salmela-Aro \& Nurmi, 1997). Las metas relacionadas con el logro de necesidades biológicas básicas predicen el bienestar en diferentes culturas. Según Pavot y Diener (1993) el tiempo libre es una de las necesidades comunes a diferentes culturas que predicen el bienestar. Las actividades relacionadas con el ocio o tiempo libre pueden ser recompensantes cuando son valoradas intrínsecamente por las personas y utilizan sus habilidades para interactuar con amigos y familia (Argyle, 1987).

El compromiso con determinadas metas está mas relacionado con la felicidad cuando esas metas son valoradas dentro de la cultura o subcultura donde el sujeto se desarrolla. La cultura influye en las metas que la gente se propone y así en las fuentes de la satisfacción (Markus \& Kitiyama, 1991; Triandis, 1989). En las culturas "individualistas", como la peruana y la española, las personas tienden a diferenciarse de los demás. Las variables relacionadas con uno mismo, por ejemplo la autoestima, se relaciona con el bienestar. En culturas "colectivistas" una de las metas del individuo es no distinguir entre uno mismo y los demás para mantener un estado de armonía. Las metas de uno mismo pueden estar subordinadas a las metas de 
Metas y satisfacción vital en adolescentes españoles y peruanos...

los demás. En estas últimas culturas, las variables relacionadas con uno mismo, como la autoestima, autoconcepto, etc., son menos importantes para determinar el bienestar que otro tipo de variables (Castro Solano \& Díaz Morales, 2000). Por ejemplo, las preocupaciones de los jóvenes norteamericanos se refieren a la finalización de los estudios, las relaciones (amigos y pareja), dinero, conflictos con los padres y "el futuro". Adolescentes canadienses indican como preocupaciones el logro social, logro académico, dinero, autorealización mediante los hobbies, salud y paz en el mundo. Los adolescentes australianos se plantean metas relacionadas con el trabajo $u$ ocupación, educación, independencia, futuro, relaciones personales y responsabilidad social. Klingman (1998) encuentra nueve dominios considerando el contenido de las metas: conductas autodestructivas, amigos, relaciones con el sexo opuesto, escuela, servicio militar, trabajo futuro, estado físico, vida social y salud. Dos de las áreas son las más relevantes: vocación futura y relaciones con el sexo opuesto. Estas categorías son específicas de un grupo de adolescentes israelíes, de ahí la preocupación por el servicio militar.

Con respecto al sexo, los resultados españoles corroboran los resultados clásicos sobre las diferencias entre varones y mujeres. Las mujeres adolescentes se plantean más metas relacionadas con los amigos, mientras que los varones adolescentes enumeran más metas relacionadas con las posesiones y cosas materiales. En el grupo peruano, la mayor presencia en las mujeres de metas relativas a la Familia y Pareja, a los Padres y al Contacto Altruista, es decir, a la preocupación por el bienestar de otros, también coincide con otros estudios donde son la mujeres las que priorizan el tema familiar. Los adolescentes varones de este estudio presentan mayor cantidad de metas de diversión, es decir, de metas relativas a actividades de ocio o a la búsqueda de placer. Estos resultados nos sugieren que en las mujeres, tanto españolas como peruanas, hay un mayor interés y preocupación por los vínculos, ya sean amicales o familiares, y en los varones predominan metas más orientadas a sí mismos como el interés en lo material o en la diversión. 
Watkins y colaboradores (1998), en un macroestudio transcultural realizado en 14 países con 3600 sujetos de 15 a 21 años, ponen de manifiesto que en las culturas que prima el individualismo frente a la colectividad siguen apareciendo diferencias de sexo, ya que las mujeres consideran más importantes los valores familiares y las relaciones sociales que los hombres (Cardenal \& Díaz Morales, 2000). En cuanto al mayor número de metas relacionadas con el contacto altruista en mujeres adolescentes, es lógico postular que esta variable estará más asociada con la autoestima en mujeres que en hombres, debido a la educación diferencial que reciben unos y otros, ya que, en este sentido, se ha encontrado en Huston (1982) y en Johnson (1975), que los padres refuerzan más en las hijas los roles de expresividad, permisividad y de ayuda a la comunidad, valorando más su valía personal cuando estas tareas son realizadas por mujeres (Cardenal, 1999).

El nivel socioeconómico presenta mayores diferencias en el grupo peruano que en el español. En este último sólo se encuentran diferencias en la cantidad de metas relacionadas con los hobbies, que se presenta mayormente en los adolescentes de NSE más alto, probablemente por un mayor acceso a oportunidades de ocio. En el grupo peruano en cambio, las diferencias se presentan en Estudios, Relación con los Padres y Contacto Altruista, en todos los casos a favor de los adolescentes de NSE bajo, que serían quienes se muestran más preocupados por estos temas. La oportunidad de estudios superiores, por ejemplo, para los jóvenes de niveles bajos es más restringida en el Perú; asimismo, estos jóvenes expresan una mayor preocupación por el bienestar o subsistencia de los Padres, frente a quienes, en muchos casos, se sienten responsables.

Cuando se considera conjuntamente la interacción entre el sexo y el NSE, se encuentra algo similar a lo descrito anteriormente. En el grupo español son las mujeres de bajo nivel socioeconómico las que se plantean un mayor número de metas relacionadas con los amigos. Además, son los varones adolescentes de alto nivel socioeconómico 
los que se plantean un mayor número de metas relacionadas con las cosas materiales. En el estudio peruano las diferencias son similares a las encontradas al considerarse el NSE independiente. Así, las metas relativas a los Estudios predominan en los hombres y mujeres de NSE bajo, y las metas vinculadas a los Padres y al Contacto Altruista predominan en las mujeres de NSE bajo.

Por otro lado, en ambos estudios no se encuentran diferencias significativas en los niveles de satisfacción. Sin embargo, las tendencias son diferentes, en el grupo español hay una mayor tendencia hacia una mayor satisfacción con la vida en las mujeres de NSE bajo; en el grupo peruano ocurre lo contrario, son las mujeres de NSE bajo las que presentan una tendencia a una menor satisfacción con la vida. La mayor satisfacción se encuentra en las mujeres de NSE alto. Estas diferencias probablemente se encuentren vinculadas a lo que supone pertenecer a un NSE bajo en ambos países. En el Perú esto significa un gran número de carencias en todos los niveles, que es lo que posiblemente marca las diferencias.

En general, podemos afirmar que en ambos estudios las metas de los adolescentes son bastante similares, lo mismo que las diferencias en torno al sexo. La variable que marca mayores diferencias entre ambos estudios es la variable socioeconómica.

\section{Referencias}

Andrews, F. \& Withey, S. (1976). Social indicators of well being: American's perceptions of life quality. Nueva York: Plenum Press.

Apoyo. (2002). Niveles socioeconómicos de la Gran Lima. Lima: Apoyo Opinión y Mercado S. A.

Argyle, M. (1987). The psychology of happiness. Londres: Methuen. Arrindell, W. A., Heesink, J. \& Feij, J. A. (1999). The Satisfaction 
with Life Scale (SWLS): Appraisal with 1700 healthy young adults in The Netherlands. Personality and Individual Differences, 26, 815-826.

Cantor, N. (1992). From thought to behavior: "Having" and "doing" in the study of personality and cognition. American Psychologist, 45(6), 735-750.

Cardenal, V. (1999). El autoconocimiento y la autoestima en el desarrollo de la madurez personal. Málaga: Algibe.

Cardenal, V. \& Díaz Morales, J. F. (2000). Diferencias según el sexo en autoestima y en su asociación con otras variables psicológicas en adolescentes. Psicología Educativa, 6(2), 105-128.

Castillo, G. (1999). El adolescente y sus retos: la aventura de hacerse mayor. Madrid: Pirámide.

Castro Solano, A. (2000). Estilos de personalidad, objetivos de vida y satisfacción vital. Un estudio comparativo con adolescentes argentinos. Tesis doctoral no publicada, Universidad Complutense de Madrid, España.

Castro Solano, A. \& Díaz Morales, J. F. (2000). Estilos de personalidad, objetivos de vida y satisfacción vital. En M. P. Sánchez-López \& M. M. Casullo (Eds.), Estilos de personalidad: una perspectiva iberoamericana. Madrid: Miño y Dávila.

Castro Solano, A. \& Díaz Morales, J. F. (2002). Objetivos de vida y satisfacción vital en adolescentes españoles y argentinos. Psicothema, 14(1), 112-117.

Dannefer, D. (1984). Adult development and social theory: A paradigmatic reappraisal. American Sociological Review, 49, $100-$ 116.

Díaz Morales, J. F. (2000). Satisfacción autopercibida en adolescentes: análisis de sus metas personales y estilos de personalidad. Memoria de licenciatura no publicada, Universidad Complutense de Madrid, España.

Díaz Morales, J. F. (2001). Diferencias intergrupales en el sistema de metas personales, satisfacción y rendimiento en adolescentes. Tesis doctoral no publicada, Universidad Complutense de Madrid, España. 
Metas y satisfacción vital en adolescentes españoles y peruanos...

Díaz Morales, J. F. \& Naveira, A. (2001). Evaluación de metas en jugadores de fútbol de categoría juvenil y absoluta. Revista de Psicología del Deporte, 10(2), 211-223.

Díaz Morales, J. F. \& Sánchez-López, M. P. (2001). Relevancia de los estilos de personalidad y las metas personales en la predicción de la satisfacción vital. Anales de Psicología, 17(2), 151158.

Díaz Morales, J. F. \& Sánchez-López, M. P. (2002). Relaciones entre estilos de personalidad y satisfacción autopercibida en diferentes áreas vitales. Psicothema, 14(1), 100-105.

Emmons, R. (1986). Personal strivings: An approach to personality and subjective well being. Journal of Personality and Social Psychology, 5l(5), 1058-1068.

Fernández, J. (1980). Clase social y nivel socioeconómico. Manuscrito no publicado, Universidad Complutense de Madrid, España.

Herrera, D. (2002). Social insertion of high school graduates in Lima. Tesis doctoral no publicada, Universidad Católica de Lovaina, Bélgica.

Huston, A. C. (1982). Sex typing. En P. H. Mussen (Ed.), Carmichael's manual of child psychology (Vol. 2) (3a. ed.). Nueva York: Wiley.

Johnson, M. M. (1975). Fathers, mothers, and sex typing. Sociological Inquiry, 45, 15-26.

Kammer, P. P. (1985). Career and life-style expectations of rural eight-grade students. The school counsellor, 33(1), 18-25.

Klingman, A. (1998). Psychological education: Studying adolescents' interests from their own perspective. Adolescence, 33(130), 435446.

Little, B. (1983). Personal projects: A rationale and a method for investigation. Environment and Behaviour, 15, 273-309.

Little, B. (1999). Personality and motivation: Personal action and the conative evolution. En L. A. Pervin \& O. John (Eds.), Handbook of personality. Theory and Research (2a. ed.) (pp. 501-524). Nueva York: Guilford Press. 
Lueptow, L. B. (1984). Adolescent sex roles and social change. Nueva York: Columbia University Press.

Markus, H. \& Kitayama, S. (1991). Culture and the self: Implications for cognition, emotion and motivation. Psychological Review, 98(2), 224-253.

Martínez, P. (2002). Perspectiva temporal futura en adolescentes de Lima-Perú. Tesis de maestría no publicada, Universidad Autónoma de Barcelona, España.

Martínez, P. (2004). Perspectiva temporal futura y satisfacción con la vida a lo largo del ciclo vital. Tesis doctoral no publicada, Universidad Autónoma de Barcelona, España.

Nurmi, J. E. (1989). Development of orientation to the future during early adolescence: A four-year longitudinal study and two crosssectional comparisons. International Journal of Psychology, 24, 195-214.

Nurmi, J. E. (1991). How do adolescents see their future? A review of the development of future orientation and planning. Developmental Review, 11, 1-59.

Nurmi, J. E., Poole, M. E. \& Seginer, R. (1995). Tracks and transitions: A comparison of adolescent future-oriented goals, explorations and commitments in Australia, Israel and Finland. International Journal of Psychology, 30, 335-375.

Nuttin, J. (1985). Future time perspective and motivation: Theory and research method. Nueva Jersey: Lawrence Erlbaum Associates.

Palys, T. \& Little, B. (1983). Perceived life satisfaction and the organization of personal projects systems. Journal of Personality and Social Psychology, 44(6), 1221-1230.

Pavot, W. \& Diener, E. (1993). Review of the Satisfaction with Life Scale. Psychological Assessment, 5(2), 164-172.

Pervin, L. A. (1989). Goal concepts and social psychology. Nueva Jersey: Lawrence Erlbaum.

Post, P., Williams, M. \& Brubaker, L. (1996). Career and lifestyle ex- 
pectations of rural eight-grade students: A second look. The $\mathrm{Ca}$ reer Development Quarterly, 44, 250-257.

Salmela-Aro, K. \& Nurmi, J. E. (1997). Goal contents, well-being and life context during transitions to university: A longitudinal study. International Journal of Behavioural Development, 20(3), 471-491.

Sheldon, K. M. \& Elliot, A. J. (2000). Personal goal in social roles: Divergences and convergences across roles and levels of analysis. Journal of Personality, 68(1), 51-84.

Shevlin, M., Brunsden, V. \& Miles, J. N. V. (1998). Satisfaction with Life Scale: Analysis of factorial invariance, mean structures and reliability. Personality and Individual Differences, 25, 911-916.

Triandis, H. (1989). The self and social behaviour in differing cultural contexts. Psychological Review, 96(3), 506-520.

Watkins, D., Akande, A., Fleming, J., Ismail, M., Lefner, K., Regmi, M. et al. (1998). Cultural dimensions, gender, and the nature of self-concept: A fourteen-country study. International Journal of Psychology, 33(1), 17-31. 